\title{
On the measurement of the generalized degree of polarization
}

\author{
J.M. MOVIL L A*, G. P I Q U E R O, P. M . M E J Í A S \\ A N D R. M A R T Í N E Z - H E R R E R O \\ Departamento de Optica, Facultad de Ciencias Físicas, Universidad Complutense, 28040 Madrid, Spain \\ (*author for correspondence: E-mail: movilla@eucmax.sim.ucm.es)
}

Received 12 November 1999; accepted 17 November 1999

\begin{abstract}
On the basis of well-known methods to obtain the standard Stokes parameters, two simple experimental procedures are analysed to measure the so-called generalized degree of polarization, $\mathbf{P}$, defined elsewhere [Opt. Commun., 149 (1998) 230]. After discussing the accuracy of the methods, the generalized degree of polarization of a non-uniformly polarized beam emerging from an optically pumped birefringent Nd:YAG rod optically pumped is experimentally investigated in terms of the pumped power.
\end{abstract}

Key words: laser beams, polarization, beam characterization, non-uniformly totally polarized beams

\section{Introduction}

As is well known (Lavi et al. 1988; Baastians 1989; Siegman 1990; Serna et al. 1991; Weber 1992), the spatial characterization of light beams by means of overall parameters has been extensively developed in the past ten years in the scalar case (also assuming homogeneous polarization across the wavefront). In practice, however, the laser beam can undergo depolarization upon propagating along optical systems containing polarizing elements or may exhibit spatial variations in its polarization state (Erdogan and Hall 1990; Lü et al. 1995; Greene and Hall 1996; Gori et al. 1998). In such cases new parameters should be introduced to describe these features. This is of particular importance with regard to the international standards that evaluate the polarization status of general (non-uniformly polarized) beams (Draft International Standard ISO/DIS 12005).

On the basis of a generalization of the Stokes-Mueller formalism to describe the intensity moments of partially polarized beams, a new overall parameter, namely, the so-called generalized degree of polarization $\mathbf{P}$, has been quite recently defined (Movilla et al. 1998). For non-uniformly totally polarized (NUTP) fields, this parameter can be understood as a measure of the uniformity of the polarization state of the field over the cross-sectional regions where the intensity is significant. The applicability and the usefulness 
of this proposal are closely connected in practice with the possibility of the accurate measurement of $\mathbf{P}$.

In the present paper, after introducing in the next section the formalism and definitions to be used, two experimental procedures to measure the parameter $\mathbf{P}$ are analysed in Section 3; the accuracy of the methods is also discussed. In Section 4, the value of parameter $\mathbf{P}$ at the output of a birefringent $\mathrm{Nd}$ :YAG rod is measured as a function of the pumping power. Finally, the main conclusions are summarized in Section 5.

\section{Formalism and definitions}

We will consider quasimonochromatic fields written as stationary random processes, propagating within the paraxial approach along the $z$-axis. From now on, we denote the associated dimensionless field vector perpendicular to the $z$-axis by

$$
\mathbf{E}(\mathbf{r}, z ; \omega)=\left(E_{s}(\mathbf{r}, z ; \omega), E_{p}(\mathbf{r}, z ; \omega)\right),
$$

where $\omega$ represents the angular frequency and $s$ and $p$ are the axes of an arbitrary orthogonal coordinate system. In Equation (1), $E_{s}$ and $E_{p}$ are the field components along these directions (note that we use in this paper $s$ and $p$, not $x$ and $y$, to describe the polarization of the field). For convenience, the spatial variables $(x, y)=\mathbf{r}$ are considered here dimensionless variables. They are defined as the product of the wavenumber, $k_{0}$, and the Cartesian coordinates of the point at which we are evaluating the field. To define the parameter $\mathbf{P}$ let us first introduce the so-called Wigner distribution function $H$ in the form (Martinez-Herrero et al. 1997; Movilla et al. 1998)

$$
H(\mathbf{r}, \boldsymbol{\eta}, z)=\frac{1}{k_{0}^{2}} \int G\left(\mathbf{r}+\frac{s}{2}, \mathbf{r}-\frac{s}{2}, z\right) \exp (\text { is } \boldsymbol{\eta}) \mathrm{d} s,
$$

where

$$
G\left(\mathbf{r}_{1}, \mathbf{r}_{2}, z ; \omega\right)=\overline{\mathbf{E}^{+}\left(\mathbf{r}_{1}, z ; \omega\right) \mathbf{E}\left(\mathbf{r}_{2}, z ; \omega\right)}
$$

is the electric field cross-spectral density matrix (the overbar symbolizes an ensemble average),

$$
\mathbf{E}^{+}=\left(\begin{array}{c}
E_{s}^{*} \\
E_{p}^{*}
\end{array}\right)
$$


is the adjoint of the field vector $\mathbf{E}$ (see Equation (1)), and $\boldsymbol{\eta}=(u, v)$, with $u$ and $v$ representing angles of propagation. For simplicity, the dependence on $\omega$ has been omitted. If we write $\mathbf{R} \equiv(x, y, u, v)=(\mathbf{r}, \boldsymbol{\eta})$, the Stokes matrices associated to a partially polarized field are defined in the form (MartinezHerrero et al. 1997; Movilla et al. 1998)

$$
S_{i}=\iint \mathbf{R}^{\mathrm{T}} \mathbf{R} \operatorname{tr}\left(\sigma_{i} H(\mathbf{r}, \boldsymbol{\eta})\right) \mathrm{d} \mathbf{r} \mathrm{d} \boldsymbol{\eta}, \quad i=0,1,2,3,
$$

where the superscript $\mathrm{T}$ indicates the transposed matrix, the symbol 'tr' denotes the trace of the matrix, $\sigma_{0}$ is the identity matrix, and $\sigma_{i}, i=1,2,3$ are the Pauli matrices. Note that matrix $S_{0}$ involves all (measurable) secondorder intensity moments of the beam.

Within the framework of the above formalism, the generalized degree of polarization $\mathbf{P}$ was recently introduced in the form (Movilla et al. 1998)

$$
0 \leq \mathbf{P} \equiv\left[\frac{\left(\operatorname{tr} S_{1}\right)^{2}+\left(\operatorname{tr} S_{2}\right)^{2}+\left(\operatorname{tr} S_{3}\right)^{2}}{\left(\operatorname{tr} S_{0}\right)^{2}}\right]^{1 / 2} \leq 1,
$$

where $\mathbf{P}=1$ for uniformly totally polarized fields. As was mentioned in the Section 1, for NUTP beams $\mathbf{P}$ was shown to represent an overall measure of the uniformity of the polarization state of the field over those transversal regions with significant intensity values.

\section{Experimental procedures to measure $P$}

The parameter $\mathbf{P}$ can be measured in several ways. Here we will propose and compare two experimental procedures to determine the traces of the Stokes matrices leading to parameter $\mathbf{P}$. Both methods involve the measurement of the total power together with the second-order intensity moments (namely, beam width and divergence) for several orientations of a polarizer and a quarter-wave plate at the observation plane.

\subsection{METHOD 1}

This method follows four steps (Movilla et al. 1998)

1. The measurements of the total power, $W$, the (squared) beam width $\left\langle r^{2}\right\rangle$ and the (squared) far-field divergence $\left\langle\eta^{2}\right\rangle$ at the observation plane when the beam freely propagates. In other words, the measurements are performed with no use of any optical polarizing element (the sharp brackets 
\langle\rangle denote the standard second-order moments defined on the basis of the well-known intensity moment formalism (Lavi et al. 1988; Baastians 1989; Siegman 1990; Serna et al. 1991; Weber 1992).

2. The measurement of the same parameters as before but now the beam passes through a polarizer which accepts linear polarizaton in the azimuth $\alpha=0^{\circ}$, where $\alpha$ shows the orientation of the transmission axis of the polarizer with respect to the $s$-axis.

3. The same measurements as in step 2 but now with $\alpha=45^{\circ}$.

4. The measurements of $W,\left\langle r^{2}\right\rangle$ and $\left\langle\eta^{2}\right\rangle$ after the beam propagates successively through a quarter-wave plate (whose fast axis makes an angle $0^{\circ}$ with the $s$-axis) and a polarizer with $\alpha=45^{\circ}$.

The resulting equations for the traces of the matrices $S_{i}$ turn out to be

$$
\begin{aligned}
& \operatorname{tr} S_{0}=4 \pi^{2}\left(k_{0}^{2}\left\langle r^{2}\right\rangle_{\mathrm{FP}}+\left\langle\eta^{2}\right\rangle_{\mathrm{FP}}\right) W_{\mathrm{FP}}, \\
& \operatorname{tr} S_{1}=\frac{1}{T_{1}-T_{2}}\left[8 \pi^{2} W_{0^{\circ}}\left(k_{0}^{2}\left\langle r^{2}\right\rangle_{0^{\circ}}+\left\langle\eta^{2}\right\rangle_{0^{\circ}}\right)-\left(T_{1}+T_{2}\right) \operatorname{tr} S_{0}\right], \\
& \operatorname{tr} S_{2}=\frac{1}{T_{1}-T_{2}}\left[8 \pi^{2} W_{45^{\circ}}\left(k_{0}^{2}\left\langle r^{2}\right\rangle_{45^{\circ}}+\left\langle\eta^{2}\right\rangle_{45^{\circ}}\right)-\left(T_{1}+T_{2}\right) \operatorname{tr} S_{0},\right. \\
& \operatorname{tr} S_{3}=\frac{1}{T_{1}-T_{2}}\left[\frac{8 \pi^{2} W_{\frac{\lambda}{4}, 45^{\circ}}}{T_{\frac{\lambda}{4}}}\left(k_{0}^{2}\left\langle r^{2}\right\rangle_{\frac{\lambda}{4}, 45^{\circ}}+\left\langle\eta^{2}\right\rangle_{\frac{\lambda}{4}, 45^{\circ}}\right)-\left(T_{1}+T_{2}\right) \operatorname{tr} S_{0}\right],
\end{aligned}
$$

where the subscript 'FP' denotes free propagation, the coefficients $T_{1}$ and $T_{2}$ are the major and minor principal transmittances of the polarizer, $T_{\hat{2}}$ is the transmittance of the quarter-wave plate. The subscripts ' 0 ', ' $45^{\circ}$ ' and ${ }^{\frac{4}{4}} \frac{\lambda}{4}, 45^{\circ}$ ' refer to the value of $\alpha$ and to the presence of the quarter-wave plate.

\subsection{METHOD 2}

In this procedure the first steps measure the total power, the beam width and the divergence after the beam crosses a polarizer oriented so as to transmit the component in the azimuths (i) $\alpha=0^{\circ}$; (ii) $\alpha=45^{\circ}$; (iii) $\alpha=90^{\circ}$; and (iv) $\alpha=135^{\circ}$. Next, the same parameters are measured after the beam propagates successively through a quarter-wave plate whose fast-axis makes an angle $0^{\circ}$ with the $s$-axis, and a polarizer with azimuth $\alpha=45^{\circ}$ and $\alpha=135^{\circ}$, respectively. Of course, the observation plane remains unchanged during the process.

It is important to note that this procedure could be shortened by excluding the two steps in which the polarizer is oriented at $\alpha=135^{\circ}$. However, this possibility has not been chosen since, in practice, it would involve higher errors: In fact, to determine, say, $\operatorname{tr} S_{3}$, we would need to take into account in the six-step procedure the moments and power corresponding to two 
orientations of the polarizer, whereas in the abbreviated method we have to use the moments and power relative to three different orientations.

Accordingly, the traces of the matrices $S_{i}$ are now given by the following equations:

$$
\begin{aligned}
\operatorname{tr} S_{0}= & \frac{4 \pi^{2}}{T_{1}+T_{2}}\left[W_{0^{\circ}}\left(k_{0}^{2}\left\langle r^{2}\right\rangle_{0^{\circ}}+\left\langle\eta^{2}\right\rangle_{0^{\circ}}\right)+W_{90^{\circ}}\left(k_{0}^{2}\left\langle r^{2}\right\rangle_{90^{\circ}}+\left\langle\eta^{2}\right\rangle_{90^{\circ}}\right)\right] \\
\operatorname{tr} S_{1}= & \frac{4 \pi^{2}}{T_{1}-T_{2}}\left[W_{0^{\circ}}\left(k_{0}^{2}\left\langle r^{2}\right\rangle_{0^{\circ}}+\left\langle\eta^{2}\right\rangle_{0^{\circ}}\right)-W_{90^{\circ}}\left(k_{0}^{2}\left\langle r^{2}\right\rangle_{90^{\circ}}+\left\langle\eta^{2}\right\rangle_{90^{\circ}}\right)\right] \\
\operatorname{tr} S_{2}= & \frac{4 \pi^{2}}{T_{1}-T_{2}}\left[W_{45^{\circ}}\left(k_{0}^{2}\left\langle r^{2}\right\rangle_{45^{\circ}}+\left\langle\eta^{2}\right\rangle_{45^{\circ}}\right)-W_{135^{\circ}}\left(k_{0}^{2}\left\langle r^{2}\right\rangle_{135^{\circ}}+\left\langle\eta^{2}\right\rangle_{135^{\circ}}\right)\right] \\
\operatorname{tr} S_{3}= & \frac{4 \pi^{2}}{T_{\frac{\lambda}{4}}\left(T_{1}-T_{2}\right)}\left[W_{\frac{\lambda}{4}, 45^{\circ}}\left(k_{0}^{2}\left\langle r^{2}\right\rangle_{\frac{\lambda}{4}, 45^{\circ}}+\left\langle\eta^{2}\right\rangle_{\frac{\lambda}{4}, 45^{\circ}}\right)\right. \\
& \left.-W_{\frac{\lambda}{4}, 135^{\circ}}\left(k_{0}^{2}\left\langle r^{2}\right\rangle_{\frac{\lambda}{4}, 135^{\circ}}+\left\langle\eta^{2}\right\rangle_{\frac{\lambda}{4}, 135^{\circ}}\right)\right] .
\end{aligned}
$$

The main difference between methods 1 and 2 arises from the existence in the former case of some measurements of the direct, freely propagating beam, without using any polarizing elements (in method 2, all the measurements involve the presence of a polarizer). As will be apparent from the following this fact can significantly affect the accuracy of the experimental determination of $\mathbf{P}$.

To test both procedures, we handle in the experiments a He-Ne laser source Spectra Physics 117 A (frequency and intensity-stabilized mode), whose polarization is known and can be assumed to be linear ( $>1000: 1)$ and uniform throughout the wavefront. The total power and the second-order moments were measured with a Newport powermeter (model 815) and with a laser beam analyser Spiricon LBA-300PC (with a CCD Pulnix TM765 camera), respectively (see Fig. 1). We use a dichroic polarizer whose extinction ratio, $T_{1} / T_{2}$, is 250 and a zero-order quarter-wave plate with $T_{\bar{\lambda}}=$ 0.98. The measured beam divergences are much smaller than the terms $k_{0}^{2}\left\langle{ }^{4}\right\rangle$, so that, in practice, they can be neglected in the two sets of Equations (7)(10) and (11)-(14).

Special care has been taken to align the system so that the beam crosses the polarizer and the quarter-wave plate through their centres. In such a case, when both optical polarizing elements rotate, their spatial inhomogeneities would have no influence on $\mathbf{P}$ because the beam would always travel through the central region.

Figs. 2 and 3 show the experimental dependence of $\mathbf{P}$ on the angle $\theta$ that forms the transmission axis of the polarizer (oriented at $\alpha=0^{\circ}$ ) with the polarization plane of the initial beam. As is clear from the figures the 


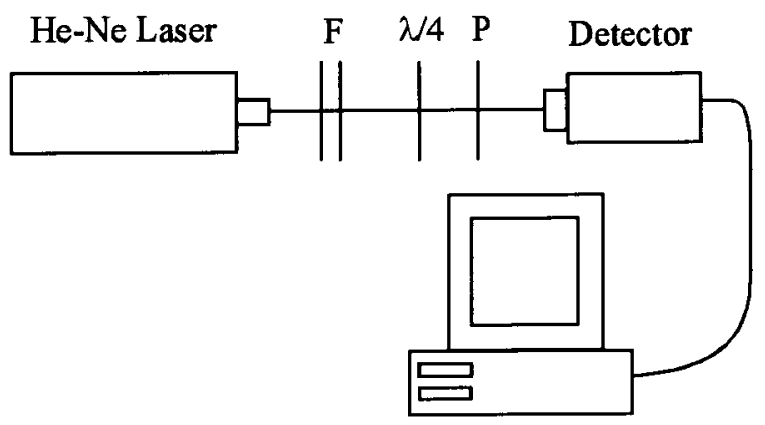

Computer

Fig. 1. Scheme of the experimental set-up used to measure the generalized degree of polarization $\mathbf{P}$ : $F$ denotes the neutral filters, $\lambda / 4$ the quarter-wave plate and $P$ the polarizer. The detector represents either the powermeter or the CCD camera.

variations of $\mathbf{P}$ with $\theta$ are smaller by using method 2. In this connection, note that, in an ideal case, $\mathbf{P}$ should remain invariant under rotation of the reference coordinate system.

On the other hand, the average value of $\mathbf{P}$ in Fig. 2 is 0.95 (method 1), whereas $\mathbf{P}$ rises up to 0.99 in Fig. 3 (according to method 2). The higher accuracy of method 2 comes from the presence of the polarizer all along the second experimental procedure. The error bars, in both figures, arise from the angular precision of the rotating mounts of both polarizing elements $\left(1^{\circ}\right)$ and, mainly, from the total power and beam widths determination. In this sense, it should be noted that, theoretically, the beam widths of a uniformly polarized beam does not change after passing through a polarizer. However,

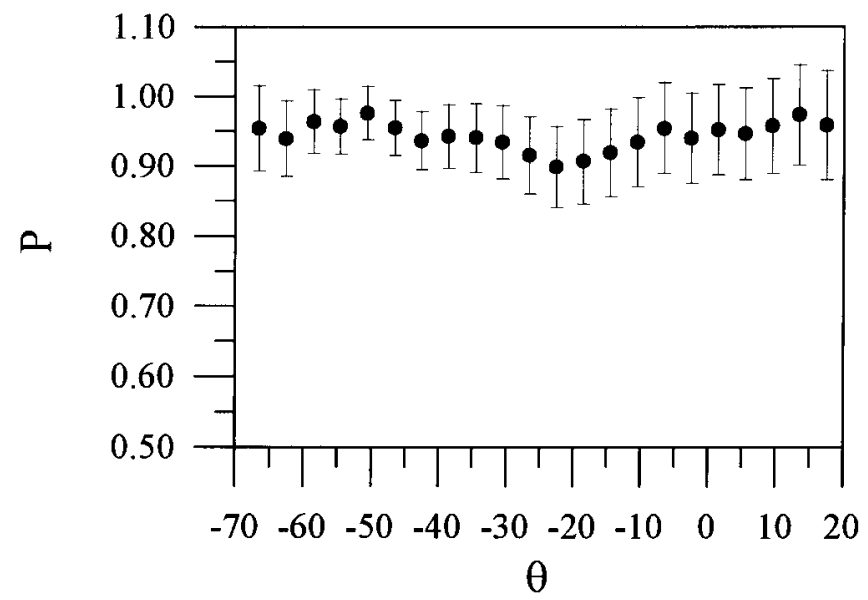

Fig. 2. Generalized degree of polarization in terms of the angle, $\theta$ (in degrees), that forms the transmission axis of the polarizer $\left(\alpha=0^{\circ}\right)$ with the polarization plane of the initial beam. $\mathbf{P}$ has been measured following method 1 . 


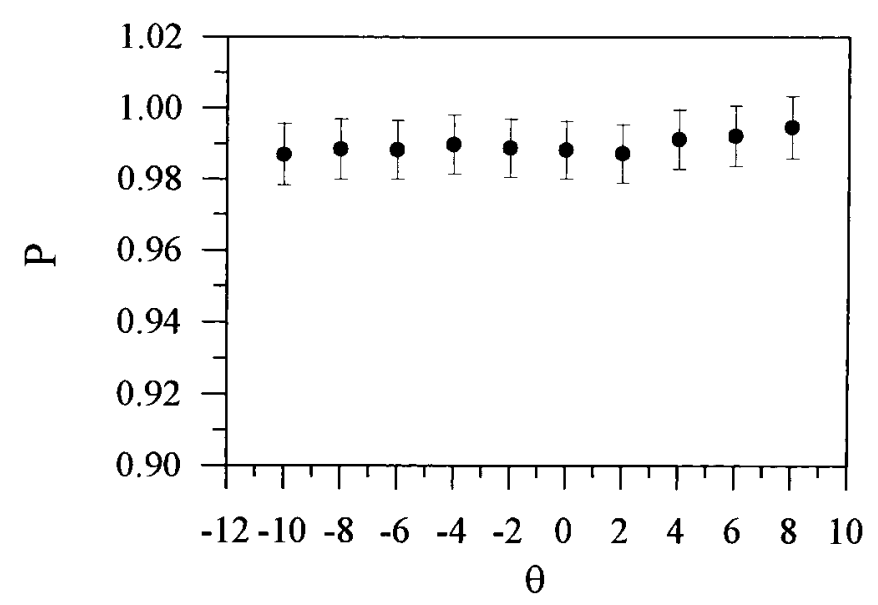

Fig. 3. Generalized degree of polarization in terms of $\theta$ (in degrees) but now $\mathbf{P}$ is measured using method 2 .

in practice, the total power reduction caused by the polarizer affects the beam size due to the influence of background and offset errors.

Taking all this into account, it seems that method 2 provides a more accurate value of $\mathbf{P}$ than method 1. In the next section we will apply that method to determine $\mathbf{P}$ of a special case of NUTP beams.

\section{Study of parameter $P$ at the output of a birefringent optically pumped Nd:YAG rod}

As is well-known the refractive index of an optically-pumped rod depends on the temperature distribution inside the material (Koechner 1976; Kugler et al. 1997). The radiation absorbed by the rod and the cooling process gives rise to a change in the refractive index with a parabolic dependence on the radial polar coordinate (assuming that the laser rod is homogeneously pumped and the heat conductivity is constant). Moreover, the temperature distribution generates different mechanical stresses along the radial and azimuthal directions so that the laser rod becomes birefringent with two different values $n_{r}$ and $n_{\phi}$ of the refractive index for light polarized along radial and azimuthal directions. The components of the refractive index may be written as (Lü et al. 1995):

$$
\begin{aligned}
& n_{r}(r)=n_{0}\left(1-\frac{\alpha_{r}}{2} r^{2}\right), \\
& n_{\phi}(r)=n_{0}\left(1-\frac{\alpha_{\phi}}{2} r^{2}\right),
\end{aligned}
$$


where $r$ represents the radial polar coordinate, $n_{0}$ is the refractive index at the centre of the rod, and $\alpha_{r}$ and $\alpha_{\phi}$ are parameters depending on a number of factors: (i) the total power supplied to the rod, $P_{i n}$; (ii) the heat efficiency, $\eta$; (iii) the rod radius, $R$; (iv) the rod length, $L$; (v) the thermal conductivity, $K_{t}$; (vi) the thermal expansion coefficient, $\alpha$; and (vii) the respective photoelastic coefficients $C_{r}$ and $C_{\phi}$ (the explicit dependence is given in Lü et al. (1995)).

Thus, when a uniformly linearly polarized beam passes through an optically pumped Nd:YAG rod, the medium introduces a non-uniform phase delay that depends on the pumping power provided to the active medium. At the output, the field becomes non-uniformly totally polarized.

We consider an incident totally polarized Gaussian beam and we assume that (i) the beam waist lies at the first rod surface; (ii) the rod has a sufficiently large aperture to admit the entire beam; and (iii) diffraction and gain saturation effects are ignored. Using the analytical expressions for the $s$ - and p-components given in Lü et al. (1995) (cf. Equations (13)) to make the computations, after lengthy but straightforward calculations we get

$$
\mathbf{P}=\frac{1}{2}\left[1+\frac{1-\beta}{(1+\beta)^{2}}\right],
$$

where

$$
\beta^{1 / 2}=\frac{n_{0}^{3} \alpha\left(C_{r}-C_{\phi}\right) w_{0}^{2}\left(\eta P_{i n}\right)}{2 \lambda K_{t} R^{2}},
$$

$w_{0}$ being the beam width.

The experimental set-up is sketched in Fig. 4. The physical parameters of the Nd:YAG rod are $L=17 \mathrm{~cm}, R=0.32 \mathrm{~cm}, n_{0}=1.82, \alpha=7.9 \times 10^{-6} \mathrm{~K}^{-1}$, $K_{t}=0.13 \mathrm{~W} \mathrm{~K}^{-1} \mathrm{~cm}^{-1}, \lambda=632.8 \mathrm{~nm}, C_{r}=0.017$ and $C_{\phi}=-0.0025$. A

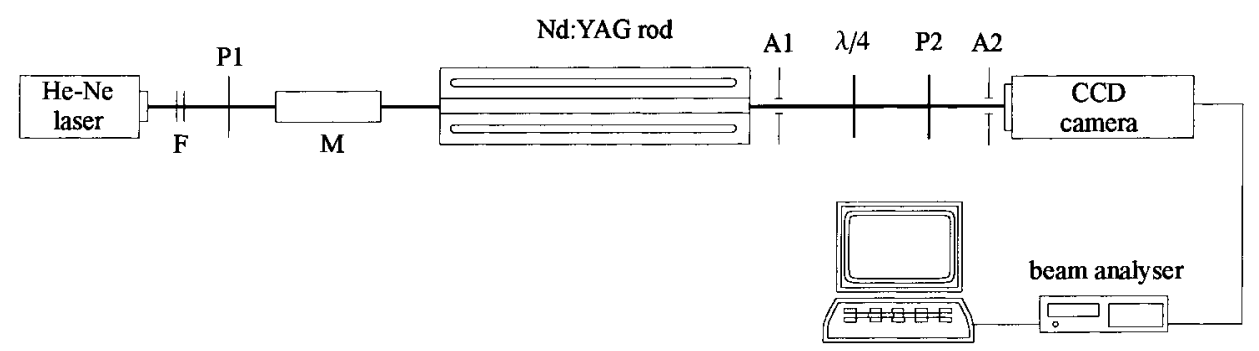

Fig. 4. Experimental set-up used to measure $\mathbf{P}$ at the output of a birefringent optically-pumped Nd:YAG rod. $F$ denotes neutral filters to attenuate the beam intensity, $P 1$ represents a polarizer that controls the azimuth of the incident beam, $M$ is a $3 \times$ magnifier that increases the beam size (it was $2 / 3$ of the radius of the rod at the entrance plane), $A 1$ and $A 2$ are two apertures to avoid the light from the pumping lamps going into the detector and, finally, $\lambda / 4$ and $P 2$ represent the quarter-wave plate and the polarizer, respectively, that are used to determine $\mathbf{P}$. 


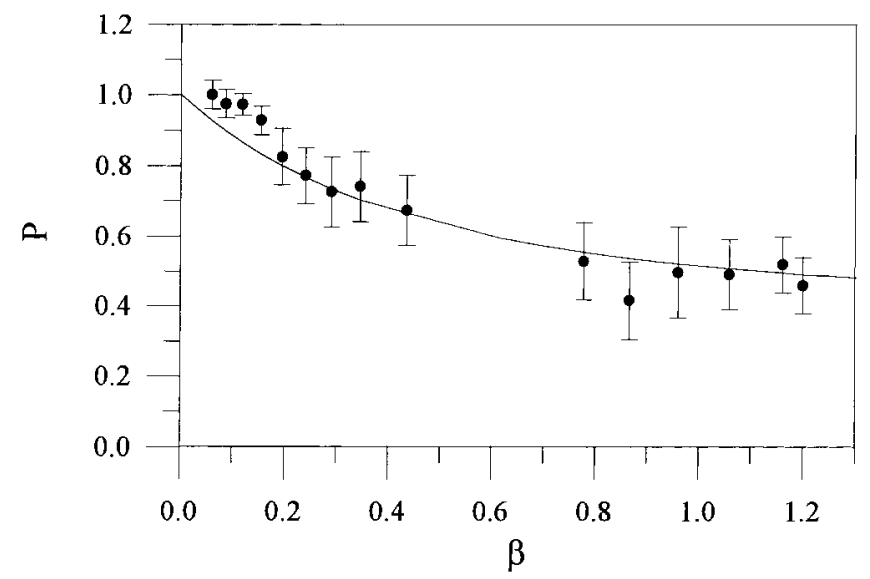

Fig. 5. $\mathbf{P}$ as a function of $\beta$ (proportional to the square of the pumping power). The dots represent the experimental values with the associated errors, and the continuous line is the theoretical curve (Equation (17)) that best fits to the experimental data. The absence of experimental values of $\mathbf{P}$ in the central region of the abcises comes from the fact that, in this interval, the beam size at the observation plane is not big enough to allow an accurate determination of the beam width. The error bars are smaller at low values of the pumping power due to the insignificant amount of light going into the CCD camera from the pumping lamps as well as the appreciable size of the beam at the observation plane for this range of $\beta$.

Kodak CCD camera Micromax model and a beam analyser are used to record and analyse the data. The laser rod was pumped by a double arc lamp up to $23 \mathrm{~A}$, and the cooling of the cavity was carried out with circulating deionised water from $40-\mathrm{kW}$ air-cooled system.

Fig. 5 plots $\mathbf{P}$ in terms of $\beta$. Note that $\beta$ is proportional to the square of the pumping power provided to the rod (cfs. Equation (18)). From the fitting procedure it follows $\eta=0.5 \%$.

As it is apparent from the figure the beam is mostly uniformly polarized over its cross-section for small $\beta$, but as $\beta$ increases, the value of $\mathbf{P}$ differs from unity, which indicates that the output field becomes a NUTP beam. This conclusion has also been inferred by other authors studying a similar system (Kugler et al. 1997). For higher values of the pumping power, $\mathbf{P}$ reaches the limit $\sim 0.5$. This fact implies the existence of a net (averaged) polarization state over the beam cross-section.

\section{Summary and conclusions}

It has been shown that the so-called generalized degree of polarization can be measured with accuracy by applying simple procedures which resembles wellknown methods to determine the standard Stokes parameters. To compare both methods we have used a uniformly linearly polarized laser source. From the experimental results it can be concluded that method 2 gives better results 
for the probe beam. Finally, we have applied the above procedure to get $\mathbf{P}$ at the output of a birefringent optically-pumped Nd:YAG rod through which a uniformly totally polarized beam propagates. The parameter $\mathbf{P}$ is expressed in terms of the pumping power.

\section{Acknowledgments}

The research work leading to this paper was supported by the Comision Interministerial de Ciencia y Tecnología of Spain, Project PB97-295. The stay of J.M. Movilla at the Technische Universität in Berlin was supported by a grant of the Universidad Complutense of Madrid. We would also like to thank Prof. H. Weber, Mr. A. Vazquez and Mr. G. Mann from the Optisches Institut at the Technische Universität in Berlin for helpful discussions as well as continuous interest and support all along the experimental work described in Section 4.

\section{References}

Baastians, M.J. Optik 82 173, 1989.

Draft International Standard ISO/DIS 12005. Test methods for laser beam parameters: Polarization. Erdogan, T. and D.G. Hall. J. Appl. Phys. 68 1435, 1990.

Gori, F., M. Santarsiero, S. Vicalvi, R. Borghi and G. Guattari. Pure Appl. Opt. 7 941, 1998.

Greene, P.L. and D.G. Hall. J. Opt. Soc. Am. A 13 962, 1996.

Koechner, W. Solid-state Laser Engineering, Springer Series in Optical Sciences, Springer, Berlin, 1976.

Kugler, N., S. Dong, Q. Lü and H. Weber. Appl. Optics 36 9359, 1997.

Lavi, S., R. Prochaska and E. Keren. Appl. Opt. 27 3696, 1988.

Lü, Q., S. Dong and H. Weber. Opt. Quantum Electron. 27 777, 1995.

Martinez-Herrero, R., P.M. Mejias and J.M. Movilla. Opt. Lett. 22 206, 1997.

Movilla, J.M., G. Piquero, P.M. Mejias and R. Martinez-Herrero. Opt. Comm. 149 230, 1998.

Serna, J., R. Martinez-Herrero and P.M. Mejias. J. Opt. Soc. Am. A 8 1096, 1991.

Siegman, A.E. Proc. SPIE 2 1224, 1990.

Weber, H. Opt. Quantum Electron. 24 1146, 1992. 